Received: 18 July 2017

Accepted: 19 October 2017

Published online: 07 November 2017

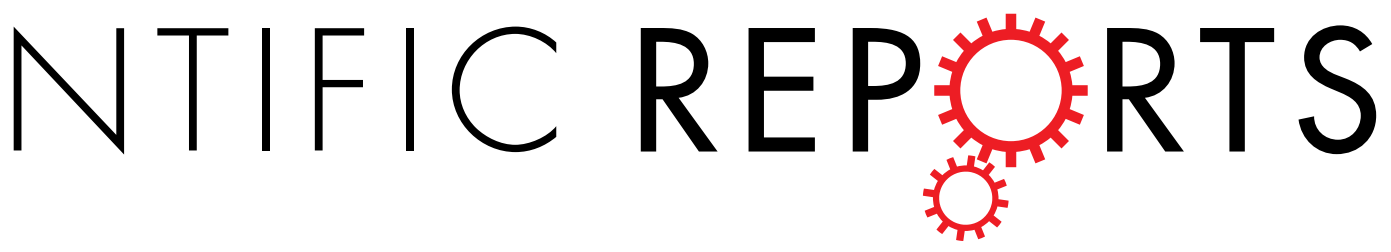

\title{
OPEN Plasmodium falciparum parasites with histidine-rich protein 2 (pfhrp2) and $p f h r p 3$ gene deletions in two endemic regions of Kenya
}

Khalid B. Beshir ${ }^{1}$, Nuno Sepúlveda ${ }^{1,2}{ }^{2}$ Jameel Bharmal ${ }^{1}$, Ailie Robinson ${ }^{1}$, Julian Mwanguzi ${ }^{1}$, Annette Obukosia Busula ${ }^{3,6}$, Jetske Gudrun de Boer ${ }^{4,7}$, Colin Sutherland $\mathbb{1}^{1}$, Jane Cunningham ${ }^{5} \&$ Heidi Hopkins ${ }^{1}$

Deletions of the Plasmodium falciparum hrp2 and hrp3 genes can affect the performance of HRP2-based malaria rapid diagnostic tests (RDTs). Such deletions have been reported from South America, India and Eritrea. Whether these parasites are widespread in East Africa is unknown. A total of 274 samples from asymptomatic children in Mbita, western Kenya, and 61 genomic data from Kilifi, eastern Kenya, were available for analysis. PCR-confirmed samples were investigated for the presence of $p f h r p 2$ and pfhrp3 genes. In samples with evidence of deletion, parasite presence was confirmed by amplifying three independent genes. We failed to amplify pfhrp2 from 25 of 131 (19.1\%) PCR-confirmed samples. Of these, only $8(10 \%)$ samples were microscopic positive and were classified as pfhrp2-deleted. Eight microscopically-confirmed $p f h r p 2$-deleted samples with intact $p f h r p 3$ locus were positive by HRP2based RDT. In addition, one PCR-confirmed infection showed a deletion at the pfhrp3 locus. One genomic sample lacked $p f h r p 2$ and one lacked $p f h r p 3$. No sample harbored parasites lacking both genes. Parasites lacking pfhrp2 are present in Kenya, but may be detectable by HRP-based RDT at higher parasitaemia, possibly due to the presence of intact $p$ fhrp3. These findings warrant further systematic study to establish prevalence and diagnostic significance.

Antigen-detecting RDTs play a key role in malaria control successes in many parts of the world, and the global availability and scale of use of RDTs has increased dramatically over the last 10 years ${ }^{1}$. Most tests currently in use detect Plasmodium falciparum histidine-rich protein 2 (HRP2) and/or plasmodium lactate dehydrogenase $(\mathrm{pLDH})$ antigens; data indicate that at least some RDT products also detect HRP3 due to a shared antigenic epitope $^{2,3}$. Compared with RDTs that detect pLDH, in general HRP2-detecting RDTs are more sensitive and are less susceptible to degradation from heat and humidity during transport and storage ${ }^{4}$. In sub-Saharan Africa, which bears $90 \%$ of the global malaria burden, RDTs accounted for $74 \%$ of diagnostic testing among suspected cases in 2015, and HRP2-based tests are the most widely used ${ }^{5}$.

Performance of HRP2-based RDTs can be affected by factors including antigenic variability of the target protein, antigen persistence in the bloodstream following elimination of parasites, and parasite density below the RDT threshold of detection ${ }^{3,6}$. In one study in western Kenya, HRP2-based RDT gave false-negative results in $5-10 \%$ and $25-30 \%$ of samples compared to microscopy and PCR respectively ${ }^{7}$. Historically such results have been ascribed to varying thresholds of detection for the different tests.

More recently, concerns have been raised that such false-negative results may reflect presence of parasites with $p f h r p 2$ gene deletions or mutations. There has long been evidence of $P$. falciparum parasites lacking the $p f h r p 2$ gene in regions of South America ${ }^{8}$, and public health authorities have always cautioned against using HRP2-based

${ }^{1}$ London School of Hygiene and Tropical Medicine, London, UK. ${ }^{2}$ Centre for Statistics and Applications of University of Lisbon, Lisbon, Portugal. ${ }^{3}$ International Centre of Insect Physiology and Ecology, Nairobi, Kenya. ${ }^{4}$ Laboratory of Entomology, Wageningen University, Droevendaalsesteeg 1, 6708 PB, Wageningen, The Netherlands. ${ }^{5} \mathrm{Global}$ Malaria Programme, World Health Organization (WHO-GMP), Geneva, Switzerland. ${ }^{6}$ Present address: Kaimosi Friends University College, Kaimosi, Kenya. ${ }^{7}$ Present address: Netherlands Institute of Ecology, Droevendaalsesteeg 10, 6708 PB, Wageningen, The Netherlands. Correspondence and requests for materials should be addressed to K.B.B. (email: Khalid.Beshir@lshtm.ac.uk) 


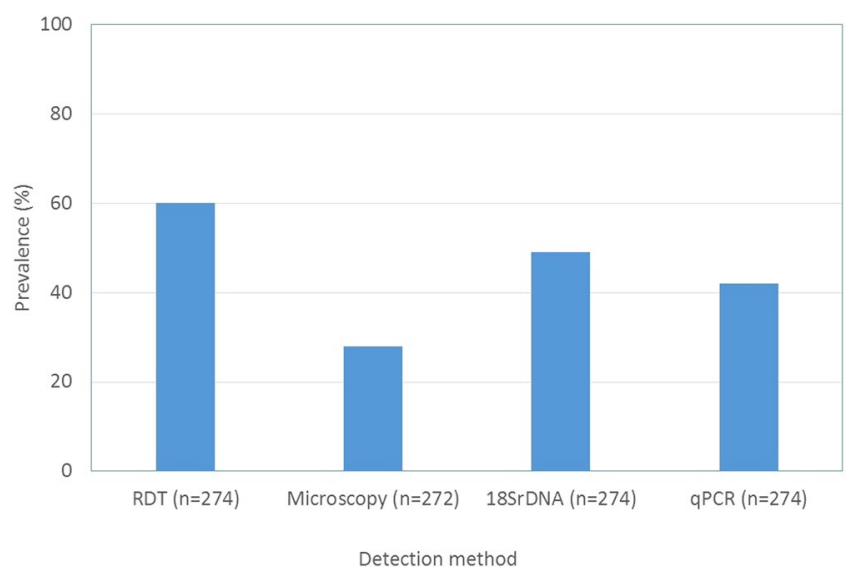

Figure 1. Proportion of different detection methods positive for malaria. Histidine-rich protein 2 (HRP2)based rapid diagnostic tests (RDTs) generated more positive samples compared to other methods. PCR methods produced $13-20 \%$ more positive results compared to microscopy.

RDTs in the Amazon River basin. In the past few years, reports have emerged of $p f h r p 2$ mutations or deletions in Africa and India ${ }^{9-12}$ leading to false-negative RDT results. More recently, in Eritrea, $80 \%$ of microscopically confirmed positive samples were negative by HRP2-based RDT, a finding attributed to deletion of the pfhrp 2 gene ${ }^{13,14}$.

The existence of parasites lacking $p f h r p 2$ would affect RDT accuracy in a broader range of malaria-endemic regions and would have significant implications for RDT implementation, clinical case management, and malaria control efforts ${ }^{15}$. Currently, there is little evidence to document the true extent of such mutations in Africa. Guidelines have recently been issued for study design and molecular assays to assess gene mutations ${ }^{16}$.

To extend current knowledge on the relationship between RDT failure and deletions of $p f h r p 2$ in African populations, we investigated samples from a cross-sectional survey in Mbita (western Kenya) and from published $P$. falciparum genome sequence data from Kilifi (eastern Kenya).

\section{Results}

Detection of $\boldsymbol{P}$. falciparum malaria. Of the 274 patient samples, $165(60 \%)$ were positive by RDT. Of these, 114 (70\%) were positive by HRP2 band only; 51 (31\%) were positive by both HRP2 and pLDH bands. Microscopy detected parasites in 76/272 (28\%) (Microscopy data were missing for two samples and of these, two (2.8\%) were RDT negative. Of the 196 microscopic negative samples, 93 (52\%) were RDT positives). The minimum parasite density reported by microscopy was 80 parasites $/ \mu \mathrm{L}$, suggesting that this was the threshold for detection by the field microscopist. Using 18SrDNA PCR and qPCR assays, 131 (47.8\%) and 114 (41.6\%), respectively, were positive for P. falciparum (Fig. 1).

PCR detection of $p f h r p 2$ and $p f h r p 3 . \quad$ In this report, the term $p f h r p 2-$ or $p f h r p 3$-negative is used for isolates that were negative by PCR for $p f h r p 2$ and/or $p f h r p 3$ in duplicate. The term $p f h r p 2$ - or $p f h r p 3$-deleted is used for isolates that met the following four criteria: microscopy positive, three independent control genes amplified (18SrDNA, $m s p 1$ and $m s p 2$ ), pfhrp2- or $p f h r p 3$-negative in duplicate, and parasite density $\geq 5 \mathrm{p} / \mu \mathrm{L}$. Pfhrp2 genotyping of samples positive by $18 \mathrm{SrDNA}$ produced $51 \%(67 / 131)$ positive and $10.6 \%(14 / 131)$ negative results. The remaining $38.2 \%$ samples (50/131) either had parasitaemia $\leq 5 \mathrm{p} / \mu \mathrm{L}$ by qPCR or were negative by $m s p 1 / m s p 2$ PCR and were excluded from further $p f h r p 2$ analysis. Therefore, only 14 of the $p f h r p 2$-negative samples were included in further gene deletion call (Fig. 2). No significant difference in multiplicity of infection was observed between $p$ fhrp2-deleted samples $(\mathrm{n}=8$, mean $=4.77$, range 3-7) and $p$ fhrp2-positive samples $(\mathrm{n}=25$, mean $=4.8$, range $3-7)$.

Pfhrp3 genotyping of samples positive by 18 SrDNA PCR gave a positive result for $71 \%(96 / 131)$. A single sample that was positive by all other criteria, including for $p f h r p 2$, was negative for $p f h r p 3$. Conversely, all 14 of the pfhrp2 negative samples were $p f h r p 3$ positive.

Association between parasitaemia and pfhrp2 PCR results. To test whether parasitaemia affected the $p f h r p 2$ and $p f h r p 3$ PCR outcome, we compared the mean parasitaemia for samples that were positive by $18 S r D N A$ and qPCR, excluding samples with parasite density $\leq 5 \mathrm{p} / \mu \mathrm{L}$ by qPCR. Mean qPCR parasitaemia for pfhrp2 positive and negative samples, respectively, was $688 \pm 923(\mathrm{n}=73$, mean $+\mathrm{SD})$ and $611 \pm 1444(\mathrm{n}=18$, mean $+\mathrm{SD}) \mathrm{p} / \mu \mathrm{L}$, a statistically significant difference (Kruskall-Wallis test, $\mathrm{P}=0.01$; Table 1 ). However, if only microscopy-positive samples are included, the difference is not significant $(n=65, p=0.48)$. The same analysis was not done for $p f h r p 3$ as the negative sample size was only one.

Confirmation of $p f h r p 2$ and $p$ fhrp3 deletions. QPCR parasite density was used to further investigate presence or absence of $p f h r p 2$ and $p f h r p 3$ genes in microscopy-negative samples. Of the total 15 samples included in the $p f h r p 2$ and $p f h r p 3$ deletion call, one was $p f h r p 2$-positive and $p f h r p 3$-negative with a parasite density of 167 $\mathrm{p} / \mu \mathrm{L}$ and negative RDT result. The remaining 14 samples were $p f h r p 2$-negative and $p f h r p 3$-positive ( $p f h r p 2-/ p$ fhrp3+); of these, $42.8 \%$ (6/14) were RDT-negative. All six of these RDT-negative samples were also negative by 


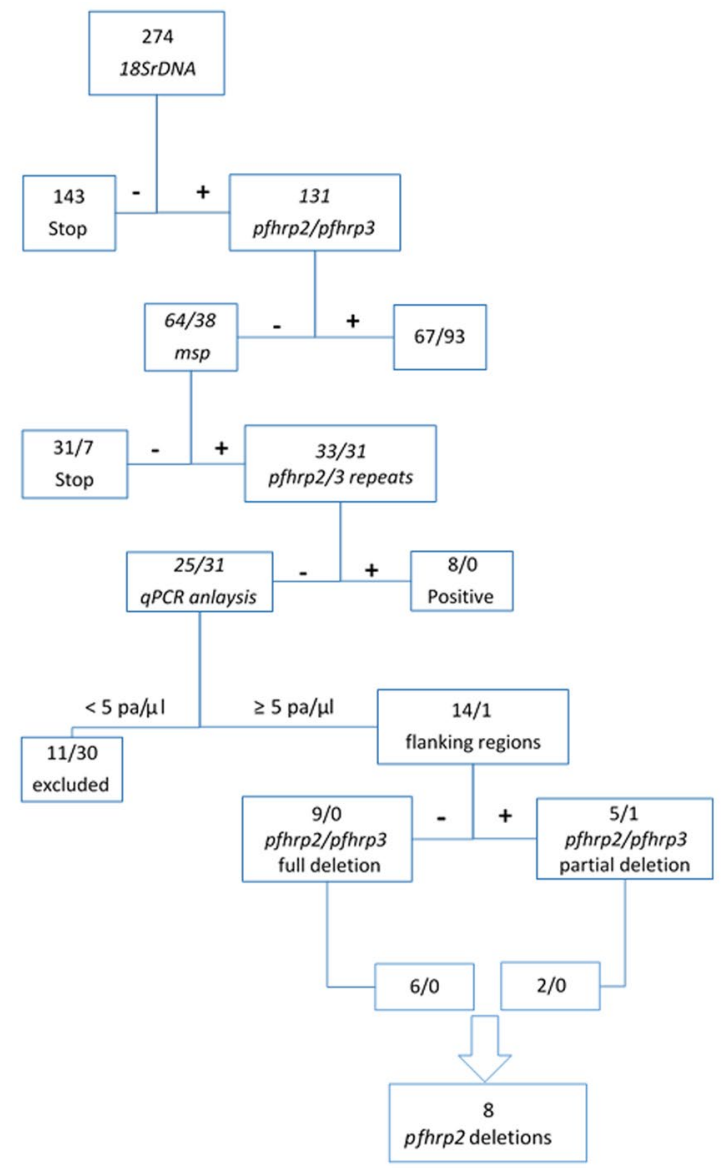

Figure 2. Flow diagram showing the strategy for determining deletion of $p f h r p 2$ and $p f h r p 3$ genes. Total of 274 Plasmodium falciparum samples were amplified using 18SrDNA PCR. All the positives (131 samples) were then subjected to $p f h r p 2$ and $p f h r p 3$ PCR amplifications. Pfhrp 2 and $p f h r p 3$ negative samples (64 and 38 respectively) were then further subjected to PCR amplification using $m s p 1$ and $m s p 2$ primers to confirm the presence of parasites. Pfhrp 2 and $p f h r p 3$ PCR amplification were repeated if the samples were $m s p 1 / m s p 2$ and $18 \mathrm{SrDNA}$ positive but $p$ fhrp2/pfhrp 3 negative (33 and 31 respectively). A qPCR analysis was then performed on those samples negative by $p f h r p 2$ and $p f h r p 3$ but positive by other PCR methods ( 25 and 31 respectively). Samples with parasitaemia less than 5 parasites per microliter were excluded ( 11 and 30 respectively). The upstream and downstream flanking regions of $p f h r p 2$ and $p f h r p 3$ for the remaining samples were then amplified using specific primers (14 and 1 respectively). To reduce the risk of false $p$ fhrp2-negative due to low parasite density, only microscopy positive samples were called $p f h r p 2$ and $p f h r p 3$ deletions (8 samples for $p f h r p 2$ only).

\begin{tabular}{|c|c|c|c|c|c|c|}
\hline & $\begin{array}{l}\text { 18SrDNA+ve } \\
N=131\end{array}$ & $\begin{array}{l}\text { 18SrDNA + ve }>5 \\
\text { parasite/ } / \mathrm{I} \mathrm{N}=91\end{array}$ & $\begin{array}{l}\text { 18SrDNA/msp1/msp2 } \\
\text { PCR }+ \text { ve }>5 \text { parasite/ul N = } 15\end{array}$ & $\begin{array}{l}\text { Mean parasite per } \mu \mathrm{L} \\
\text { (range) by } \text { qPCR }^{1}\end{array}$ & $\begin{array}{l}\text { Mean parasite/ } \mu \mathrm{L} \\
\text { by microscopy }^{1}\end{array}$ & P-value ${ }^{2}$ \\
\hline pfhrp2+RDT - & $1(0.8 \%)$ & $1(1.1 \%)$ & 1 & 167 & 0 & $0.01^{2 a} 0.48^{2 b}$ \\
\hline pfhrp2+RDT + & $74(56.4 \%)$ & $72(79.1 \%)$ & NA & 689 (8.2-3923) & $1317(0-7840)$ & \\
\hline pfhrp2-RDT+ & $34(26 \%)$ & $10(11 \%)$ & 8 & $1088(8.5-6097)$ & $1540(240-3560)$ & \\
\hline pfhrp2-RDT- & $22(16.8 \%)$ & $8(8.8 \%)$ & 6 & $10(1.2-28.1)$ & 0 & \\
\hline
\end{tabular}

Table 1. Correlation between pfhrp2 PCR result and Plasmodium falciparum parasitaemia. ${ }^{1}$ For samples that were positive by both $18 S r R N A$ and qPCR, with qPCR parasitaemia $\geq 5$ parasites per $\mu \mathrm{L}{ }^{2}$ Kruskal-Wallis rank test for ${ }^{2 a} p f h r p 2+$ and $p f h r p 2-$ of all samples, and ${ }^{2 b}$ for samples with microscopically detected parasitaemia (i.e. excluding samples with submicroscopic parasitaemia). S18rDNA, 18 ribosomal RNA subunit gene; $p$ fhrp2 and pfhrp3, Plasmodium falciparum histidine-rich protein 2 and 3 respectively; RDT, rapid diagnostic test.

microscopy suggesting that the parasitaemia was sub-microscopic. Fifty-seven per cent (8/14) of the $p f h r p 2-/ p$ fhrp3+ and RDT-positive (either HRP only, or both HRP and pLDH) samples were also positive by microscopy with a mean density of $1540 \pm 1123 \mathrm{p} / \mu \mathrm{L}$ and mean relative parasite density by qPCR of $1358 \pm 1680$. Therefore, $9 \%(8 / 89)$ of samples with adequate parasitaemia as measured by both microscopy and qPCR were $p$ fhrp2-deleted (Table 2). In addition, 6.7\% (6/89) and 1.1\% (1/89) of qPCR-confirmed samples were $p f h r p 2$ and $p f h r p 3$-negative, 


\begin{tabular}{|l|l|l|l|l|l|l|l|l|}
\hline Isolate & RDT & Parasitaemia & PF3D7_0831900 & pfhrp2 & PF3D7_0831700 & PF3D7_1372100 & pfhrp3 & PF3D7_1372400 \\
\hline K165 & + & 3560 & - & - & + & + & + & + \\
\hline K027 & + & 240 & - & - & - & + & + & + \\
\hline K065 & + & 1720 & - & - & - & + & + & + \\
\hline K031 & + & 2400 & - & - & - & + & + & + \\
\hline K063 & + & 2120 & - & - & - & + & + & + \\
\hline K233 & + & 840 & - & - & - & + & + & + \\
\hline K263 & + & 480 & - & - & - & + & + & + \\
\hline K320 & + & 960 & - & - & + & + & + & + \\
\hline K182 & - & $0 *$ & + & + & + & - & - & + \\
\hline
\end{tabular}

Table 2. Plasmodium falciparum samples with $p f h r p 2$ or $p f h r p 3$ deletion. Nine samples positive by three independent PCR assays, two of which target single-copy genes, were negative by PCR for $p f h r p 2$ or $p f h r p 3$. PCR outcome of the corresponding flanking regions are also shown. *Positive by qPCR with parasitaemia of 167 parasites per $\mu \mathrm{L}$. Pfhrp 2 and $p$ fhrp3, Plasmodium falciparum histidine-rich protein 2 and 3 respectively; RDT, rapid diagnostic test; PF3D7_0831900 and PF3D7_0831700, upstream and downstream flanking regions of $p f h r p 2$, PF3D7_1372100 and PF3D7_1372400, upstream and downstream flanking regions of $p f h r p 3$.

respectively. All the $p f h r p 2-/ p f h r p 3+$ samples were positive by three separate PCR assays, two of which targeted single-copy genes (18sDNA, msp1 and msp2).

Relationship between RDT and parasitaemia of pfhrp2-Ipfhrp3+ parasites. To explore the possibility that differences in parasitaemia of the $p f h r p 2-/ p f h r p 3+$ samples affect RDT results, samples were categorized by RDT result. Those $p f h r p 2-/ p f h r p 3+$ samples that were RDT-positive had significantly higher parasite density (mean $+\mathrm{SD}, 1358 \pm 1980 \mathrm{p} / \mu \mathrm{L}$ ) compared to the RDT-negative samples (mean $+\mathrm{SD}, 17 \pm 9, \mathrm{p}=0.0014)$. However, with available data it is not possible to determine whether these RDT were negative due to absence of $p f h r p 2$ or due to low HRP2 antigenemia resulting from low parasitaemia. To extend this analysis, generalized linear models including parasitaemia and $p f h r p 2 / p f h r p 3$ status as covariates were fit to the data from field samples with parasitaemia $\geq 5 \mu \mathrm{l}$ (See Supplementary Table 1). The best model for the data was based on complementary log-log regression and it would appear to describe the data well (Hosmer-Lemeshow goodness-of-fit test, $\mathrm{p}=0.90$ ) and a good prediction power (area under the receiver operating characteristic curve $=0.96 ; 95 \%$ $\mathrm{CI}=(0.92-1))$. According to this model for the data, the probability of RDT positivity for $p f h r p 2-/ p f h r p 3+$ samples increased with parasitaemia and was close to the one for $p f h r p 2+/ p f h r p 3+$ samples for parasitaemia levels $>1000 \mu \mathrm{l}$ (Fig. 3). Such proximity of the two probabilities together with the reduced number of $p f h r p 2-/ p f h r p 3+$ samples implied that they were not statistically different as function of parasitaemia (effect of HRP3 $=1.09$, $\mathrm{SE}=0.73, \mathrm{p}=0.14 ;$ Supplementary Table 1 ).

Analysis of $p f h r p 2$ and $p f h r p 3$ genes using genomic data from Kilifi. According to the cutoff values for coverage in Fig. 4 ( 0.7 and 0.65 cutoff values for genome with coverage and mapped reads respectively), 12 of 61 genomes could not be mapped onto the 3D7 reference genome (Fig. 4A), and thus they were discarded from further analysis.

Among the 49 remaining genomes, only two showed a high percentage ( $>50 \%)$ of genomic positions with zero coverage in $p f h r p 2$ and/or $p f h r p 3$ genes (Fig. 4B). Data from one of these genomes revealed no coverage for the entire $p f h r p 2$ locus with the exception of coverage provided by a single read mapped onto the left flanking region, providing evidence for a possible deletion of this gene (Fig. $4 \mathrm{C}$ ). In contrast, the $p f h r p 3$ locus shows some variations in coverage but mostly in a good agreement with the average coverage over the whole genome. This result suggests that the lack of coverage observed in the $p$ fhrp2 locus is unlikely to have resulted from problems in mapping these genes. Data from the other genome showed a possible deletion of the $p f h r p 3$ gene with only three reads mapped onto its extended locus (Fig. 4D). Given the good agreement between the coverage observed in the $p f h r p 2$ coding regions and the overall average coverage, the existence of three mapped reads onto the $p f h r p 3$ gene could have simply resulted from a mapping artifact due to sequence homology between the two genes. Finally, there is no evidence for the presence of genomes with possible deletions in both genes (Pearson-Clopper $95 \%$ confidence interval for the respective proportion: $0-7.3 \%)$.

\section{Discussion}

This study provides evidence of $P$. falciparum lacking the $p f h r p 2$ gene in two areas of Kenya. Eight of 89 (9\%) samples analyzed from western Kenya were $p$ fhrp2-deleted; and genomic data from eastern Kenya identified a genome with no coverage for the $p f h r p 2$ gene or flanking regions. In addition, we have confirmed that parasite density is a factor both for PCR detection of $p f h r p 2$ and for determining RDT outcome in samples lacking $p f h r p 2$, particularly in samples with submicroscopic parasitaemia. Of note, the microscopically-confirmed $p f h r p 2$-deleted samples were positive by HRP2-based RDTs. This is surprising as previously reported parasites lacking $p f h r p 2$ were generally negative by HRP2-based RDTs regardless of $p f h r p 3$ status $2,8,10,17$ though some reported positive RDT results ${ }^{8,18}$.

The $p f h r p 2$-negative samples included in this deletion call had microscopy-confirmed $P$. falciparum density of at least $250 \mathrm{p} / \mu \mathrm{L}$. The integrity of the DNA in the samples and exclusion of PCR inhibitors was verified by successful amplification using three separate PCR assays, two of which targeted single-copy genes. One other possible 


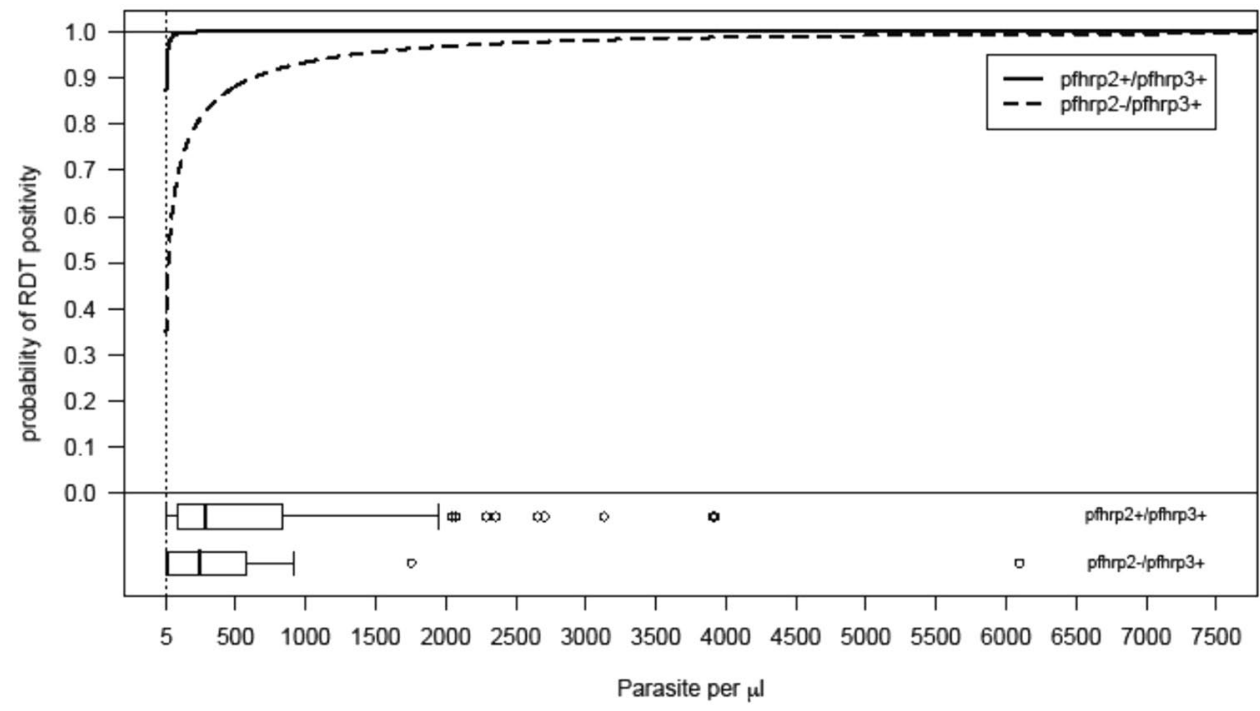

Figure 3. Complementary log-log regression model for the probability of RDT positivity as function of parasitaemia and $p f h r p 2 / p f h r p 3$ status in field samples from western Kenya with parasitaemia $\geq 5$ parasite per $\mu \mathrm{l}(\mathrm{n}=91)$ : The model shows the probability of RDT positivity in samples where both $p f h r p 2$ and $p f h r p 3$ genes are present ( $p f h r p 2+/ p f h r p 3+$, solid line) and in samples with $p f h r p 2$ deletion ( $p f h r p 2-/ p f h r p 3+$, dashed line) across a range of parasitaemia ( $p f h r p 2-/ p f h r p 3$ - not included in the model as there were no such profile in the samples). The corresponding boxplots of parasitaemia as function of $p f h r p 2 / p f h r p 3$ status are also shown at the bottom of the plot.

cause for $p f h r p 2$ - or $p f h r p 3$-negative is sequence variability of the region where the primers bind. However, the upstream and downstream segments of $p f h r p 2$ also gave negative results for at least six samples. Possible conclusions are that either these HRP2-based RDT results were false-positive, or the HRP signal was generated by protein expressed from $p f h r p 3$ or other genes; the latter explanation seems more plausible as explained below.

These results are in agreement with an in vitro study showing that the D10 parasite clone, which lacks $p f h r p 2^{19}$, gave positive RDT results on two HRP2-based RDT at parasitaemia $\geq 1000 \mathrm{p} / \mu \mathrm{L}^{8}$. Six of the eight RDT-positive Kenyan samples with $p f h r p 2$ deletion had parasitaemia $\geq 890 \mathrm{p} / \mu \mathrm{L}$ (mean, 1358), which supports the in vitro finding. Sequence analysis of HRP2 and HRP3 revealed that they share similar alanine- and histidine-rich amino acid repeats and that four of the eight repeats found in HRP 3 are identical to those of HRP $2^{2}$. It is possible that antibodies raised against HRP2 cross-react with epitopes of HRP3 to generate an RDT signal, as previously reported ${ }^{20}$. However, the level of HRP3 contribution to the signal is unknown. It is also not clear whether the HRP3 expressed is enough to generate signal in the absence of HRP2 expression or if other proteins are involved, as Baker et al. observed no compensatory increase of $p f h r p 3$ transcription in the absence of $p f h r p 2^{21}$. However, a study using yeast knockout collections reported that deletion of individual genes in a genome results in a genomic disturbance capable of driving the selection for mutations elsewhere in the genome ${ }^{22}$. The loss of $p f h r p 2$ may drive selection for mutations in $p f h r p 3$ or other similar genes particularly in the alanine- and histidine-rich amino acid repeats. This could be established by sequencing $p f h r p 3$ in parasites with and without $p f h r p 2$ and determining whether there is any sequence or repeat pattern difference. Overall, the genotypic data presented here together with the in vitro study elsewhere ${ }^{8}$ show that parasites with the $p f h r p 2-/ p f h r p 3+$ genetic profile can produce a positive result on at least some brands of HRP2-based RDTs.

Genomic data from eastern Kenya samples also showed evidence for parasites with possible deletions in $p f h r p 2$ or $p f h r p 3$ genes. If the detected deletions are true, it is difficult to infer whether they existed at the time of original infection, or resulted from a subsequent laboratory adaptation. Illumina short read sequencing used to generate the genomic data also poses a limitation of this study because of its propensity to generate uneven coverage profiles across the genome (e.g., high coverage at certain loci and little to no coverage at other loci) thus possibly influencing the probability of correctly detecting possible gene deletions. The origin of such coverage heterogeneity can be due simply to library preparation and sequencing bias of smaller fragments. The highly AT-enriched P. falciparum genome also is known to generate biases in the coverage distribution ${ }^{23}$. In theory, the use of alternative sequencing platforms that are able to generate longer reads (i.e., pacbio or minION) could produce higher quality coverage data either by mapping onto the 3D7 reference genome or by de novo assembly of the sampled genomes. In practice, this study used publicly available genomic data generated elsewhere and, therefore, it was not possible to perform alternative genomic approaches. However, the exclusion of genomic samples with sufficiently large proportions of regions with zero coverage minimized the chance of inferring deletions in the $p f h r p 2$ and $p f h r p 3$ loci when there are in fact none. A limitation of our analysis is the possibility of underestimating the true number of deletions due to challenges in mapping these genes. Pfhrp 2 and $p f h r p 3$ genes share high sequence homology; therefore mapping algorithms such as the bwa-mem, used in the pre-processing of the samples, usually allocate reads randomly to different homologous sequences present in the genome. If this is the case, 

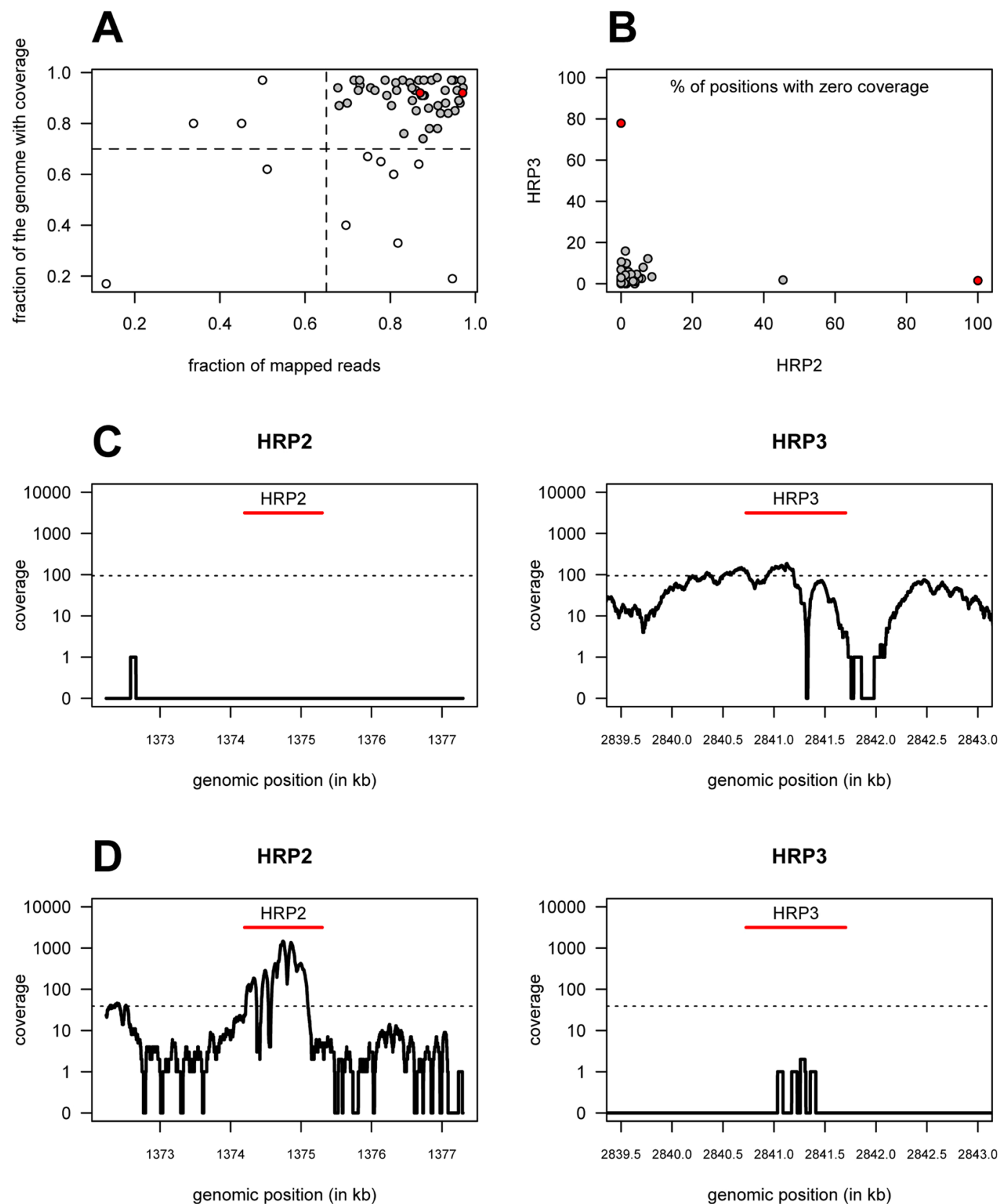

Figure 4. Coverage analysis of $p f h r p 2$ and $p f h r p 3$ genes using whole genome sequencing data from Kilifi, eastern Kenya. (A) Scatterplot of the percentage of genome with coverage (i.e., percentage of positions with at least one read mapped) versus percentage of reads that could be mapped on the 3D7 reference genome before quality checks $(n=61)$. Vertical and horizontal lines indicate the quality control cut-off used in the analysis while grey and white dots represent the genomes selected and not selected for the subsequent coverage analysis. (B) Scatterplot of the percentage of positions of $p f h r p 2$ and $p f h r p 3$ genes with no coverage after quality checks $(\mathrm{n}=49)$. Red dots in A and B represent samples with possible evidence for a deletion in either gene. (C and D) Coverage profiles of the $p f h r p 2$ and $p f h r p 3$ genes and their respective $2 \mathrm{~kb}$ flanking regions to the either side for two genomes with possible evidence for a deletion in either gene, where dotted lines represent the average coverage of the whole genome in the respective samples.

however, the existence of a deleted $p f h r p 2$ gene in presence of fully functional $p f h r p 3$, although limiting the power of genomic analysis, is unlikely to have strong consequences in the RDT testing as shown by our model (Fig. 3).

These data also confirm the role of parasitaemia in generating RDT signal by the $p f h r p 2-/ p f h r p 3+$ parasites. The model shows that parasites lacking $p f h r p 2$ produce positive HRP test bands at similar parasitaemia to those with the intact gene particularly at higher parasitaemia, supporting a previous report of the absence of compensatory increase of the HRP3 protein ${ }^{21}$. This was evident on the six $p$ fhrp2-negative samples, which were also negative by RDT and microscopy but positive for $P$. falciparum by qPCR at very low parasitaemia. We did not identify 
these parasites as lacking $p$ fhrp 2 since the negative result was more likely due to low parasitaemia, and a criterion for establishing presence of deletion is the confirmation of parasites by microscopy ${ }^{16}$. We identified the only sample with $p$ fhrp3-negative result as deletion, as the sample had adequate parasitaemia by qPCR, and microscopy might have been false negative as the slide was only read once. Further study of samples with adequate levels of parasitaemia will help to confirm these findings.

The sensitivity and clinical performance of HRP2-based RDTs in Kenya are not expected to be affected by the findings of this study. In this study, HRP2-based RDT results were positive in the presence of $P$. falciparum infection regardless of $p f h r p 2$ gene status, particularly at higher parasitaemia (above approximately $1360 \mathrm{p} / \mu \mathrm{L}$ ), presumably due to antigen-antibody cross-reactivity with HRP3 or other proteins. In addition, parasitaemia tends to be higher in symptomatic patients and therefore the effect of $p f h r p 2$ deletion on patient diagnosis is expected to be even lower. Reassuringly, a recent study that evaluated eight different HRP2-based RDTs among 500 symptomatic and asymptomatic individuals in western Kenya, and reported sensitivity compared with microscopy of $90-95 \%{ }^{7}$.

These data illustrate the difficulty of amplifying $p f h r p 2$ and $p f h r p 3$ genes in samples with submicroscopic parasite density, and support the guidance to rule out low parasitaemia as the cause of negative PCR results before reporting $p f h r p 2$ and $p f h r p 3$ deletions ${ }^{16}$. These results also highlight that to determine the true prevalence of pfhrp2 deletions in the population, the surveys should include HRP2 RDT-positive, as well as HRP2-RDT negative samples. Furthermore, investigations should include PCR testing for both $p f h r p 2$ and $p f h r p 3$, because this may be the most accurate way to estimate the risk of false negative RDT samples due to $p f h r p 2$ deletions in clinical settings. A limitation of this study is lack of HRP ELISA data to quantify HRP antigenemia and further PCR findings. The difference between $p f h r p 2+/ p f h r p 3+$ and $p f h r p 2-/ p f h r p 3+$ samples particularly at lower parasitaemia could be due to difference in the amount of antigens generated and in relative affinity of HRP 2 and HRP 3 for the RDT antibodies, which we could not measure and which our model (Fig. 3) did not take into account. This study is also limited in that the genomic and genotype data are not from the same samples or from the same area in Kenya, although malaria endemicity in both regions is similar ${ }^{24}$.

In summary, results of this study demonstrate that $P$. falciparum isolates lacking $p f h r p 2$ genes are present in Kenya, they infect humans, and cause symptomatic disease but they are also still detectable by at least some brands of HRP2-based RDTs in the presence of an intact $p f h r p 3$ expressing antigen levels found at parasitaemia $\geq 1000 \mathrm{p} / \mu \mathrm{L}$ However, these findings warrant further systematic study to understand factors driving $p$ fhrp 2 deletions; to establish the true prevalence and distribution of $p f h r p 2$ and $p f h r p 3$ deletions and to monitor for false negative HRP2 based RDTs and the potential implications for diagnostic testing strategies.

\section{Materials and Methods}

Study populations. A total of 274 blood samples were collected between January and July 2014 from asymptomatic children aged 5 to 12 years attending schools near Mbita, western Kenya, as part of a study on mosquito behavior and the odor profile of malaria infected individuals. The study received approval from the Kenya Medical Research Institute Ethical Review Committee (KEMRI/RES/7/3/1) and the London School of Hygiene and Tropical Medicine Ethics Committee (reference 8510). Written informed consent was obtained from a parent or guardian of each participating child. All experiments were performed in accordance with relevant guidelines and regulations.

Data from 61 P. falciparum samples from Kilifi District, eastern Kenya, were obtained from the MalariaGEN Plasmodium falciparum Community Project as described in Genomic epidemiology of artemisinin resistant malaria $^{25}$. These genomic data were generated from parasite samples which were obtained from pediatric patients enrolled in a clinical trial as described elsewhere, and then cultured in the lab until full adaptation ${ }^{26}$.

Blood sample preparation of the Mbita samples. Finger-prick blood from each participant was analyzed by light microscopy for the presence of parasites; applied to an RDT (SD Bioline Malaria Ag P.f/Pan test, HRP2/pan-pLDH, catalog number 05FK60, Standard Diagnostics, Hong Dong, Korea); and used to prepare dried blood spots (DBS) which were stored at room temperature until they were shipped to London School of Hygiene \& Tropical Medicine (LSHTM). Slides were read by a study team microscopist trained at the Walter Reed Project in Kisumu, Kenya, and a subset (10\%) of slides were re-read independently by a second microscopist at the LSHTM Malaria Reference Laboratory, London, UK. For the double-read subset, correlation between the two readers was moderate $(r=0.76, P<0.001)$. RDTs were prepared and interpreted by a study team member according to the manufacturer's instructions.

The RDTs and DBS were sent to LSHTM for molecular analysis. RDTs were available for all 274 samples, while DBS were available for 141. (Investigators for the primary entomology study modified their protocol to include DBS only midway through the study). Both template types were available for 141 samples, which were used as a comparison to validate the extraction of DNA from RDTs and subsequent quantification of parasitaemia by qPCR, with no significant difference observed (A Robinson, in preparation).

DNA was extracted using a robotic extraction system (QIAsymphony) using DSP DNA mini kit according to the manufacturer's protocol (QIAGEN, Germany). In order to reduce costs, we validated the DSP DNA mini kit instead of using the manufacturer-recommended investigator kit for DNA extraction from DBS and RDTs with no significant difference (A Robinson, in preparation). DBS were pre-treated according to the manufacturer's manual before DNA extraction. Briefly, $1 \times 3 \mathrm{~mm}$ diameter punch from the DBS or three cuts of the nitrocellulose strip of the $\mathrm{RDTs}^{27}$ were placed in the deep-well plate. Buffer ATL $(180 \mu \mathrm{L})$ and proteinase $\mathrm{K}(20 \mu \mathrm{L})$ were added to each well and mixed by thermomixer at $900 \mathrm{rpm}$ at $56^{\circ} \mathrm{C}$ for $15 \mathrm{~min}$. The deep-well plate was then placed directly into the sample compartment of the QIAsymphony for DNA extraction. The extracted DNA samples were stored at $-20^{\circ} \mathrm{C}$ for further molecular biology investigation. 
Amplification of $p f h r p 2$ and $p f h r p 3$. The presence of parasites was detected by standard PCR targeting the $18 \mathrm{~S}$ ribosomal RNA gene of $P$. falciparum (18SrDNA), which has a limit of detection of 0.1 parasite per $\mu \mathrm{L}$ $(0.000002 \% \text { parasitaemia })^{28,29}$. All samples positive by $18 \mathrm{SrDNA}$ assay were then subjected to $p$ fhrp 2 and $p$ fhrp3 genotyping using previously published PCR conditions and primers as described by Baker et al.$^{2,8}$. The elongation temperature was modified to $60^{\circ} \mathrm{C}$ and annealing temperature to $50^{\circ} \mathrm{C}$ in the repeated experiments of $p$ fhrp 2 and pfhrp3 (Fig. 2) $)^{11}$.

Confirmation of $p f h r p 2$ and $p f h r p 3$ deletion. To verify the presence of parasites in the $p f h r p 2$-negative samples, PCR assays targeting $m s p 1$ and $m s p 2$ genes were amplified using previously reported methods ${ }^{30}$. Microscopy-positive samples with positive results for $18 \operatorname{SrDNA}, m s p 1$ and $m s p 2$ assays but negative results for $p f h r p 2$ / pfhrp3 were considered $p f h r p 2$ / $p f h r p 3$-deleted after excluding low parasitaemia in order to avoid incorrect deletion calls (See Fig. 2 for algorithm).

Parasitaemia estimation by qPCR. To explore the role of low parasitaemia in apparently negative $p$ fhrp2 Ipfhrp3 genotyping results, the parasitaemia of each sample was quantified using a previously published PgMET qPCR assay, which has a limit of detection of 5 parasites per $\mu \mathrm{L}^{31,32}$. All samples, including samples that were negative by $18 \mathrm{SrDNA}$ assay, were included in order to capture any false negatives. International Standard was used as a positive control (calibrator) with the original sample (9.6\% parasitaemia) diluted to $0.0096 \%$ ( 500 parasite per $\mu \mathrm{L})^{33}$. The accuracy of this quantification was then compared to parasitaemia generated by microscopic examination of blood films, when available.

Statistical analysis of field samples. The main outcomes for this study were results of HRP-based RDT, results of $p f h r p 2$ and $p f h r p 3$ PCR, and parasitaemia as determined by microscopy and qPCR. Parasite density measured by qPCR for each sample was calculated using International Standard (IS) as a calibrator ${ }^{32,33}$, which was used as factor to calculate the parasitaemia of each sample. Parasitaemia was compared between groups (e.g. RDT-positive vs RDT-negative samples, $p$ fhrp2-positive vs $p f h r p 2$-negative) using Kruskal-Wallis rank test. Samples below the qPCR limit of detection $(\leq 5$ parasites per $\mu \mathrm{L}[\mathrm{p} / \mu \mathrm{L}])$ were excluded from further $p$ fhrp 2 and $p f h r p 3$ deletion call. Generalized linear models for binomial responses were used to study the probability of RDT positive with log parasitaemia, $p$ fhrp2/pfhrp3 status, as described in S1 Table. The best model was selected according to the Akaike's and Bayesian Information Criteria. The statistical validation of this model was made via the Hosmer-Lemeshow goodness-of-fit test using 5 bins to calculate the quantiles. The area under the curve (AUC) of the receiver operating characteristic curve predicted by the model was also estimated in order to assess the predictive power of the model. For the Hosmer-Lemeshow test, a p-value $>0.05$ indicated a good fit of the model while an estimate of the AUC close to 1 provided evidence for a very good prediction of the observed RDT positivity data by the model.

Data analysis was performed using either STATA (version 14, USA), or R (version 3.3.2, http//www.r-project. org). In the R software, the packages ResourceSelection and pROC were specifically used to perform the Hosmer-Lemeshow test and to estimate AUC, respectively.

Analysis of pfhrp2 and pfhrp3 genes using whole-genome sequencing data. The 61 P. falciparum samples from Kilifi underwent whole-genome sequencing and were processed as described in detail elsewhere ${ }^{34}$. Briefly, for each sample, the raw sequence reads were aligned onto the 3D7 reference genome (version 3.0) using the bwa-mem short read alignment algorithm. For each sample, the total number of mapped reads per position (coverage) was calculated for the whole genome. The genomic analysis of $p f h r p 2$ and $p f h r p 3$ genes (PF3D7_0831800 and PF3D7_1372200) was restricted to position 1,372,236-1,377,299 of chromosome 8 ( $p$ fhrp2 locus), and to position 2,838,727-2,843,703 of chromosome 13 ( $p f h r p 3$ locus). These two genomic regions included the coding regions of each gene and the respective $2 \mathrm{~kb}$ flanking regions (to either side). The corresponding coverage data was then analyzed to identify samples with possible deletions of these genes, using a statistical description of the coverage data. The application of existing genomic tests for detecting deletions using coverage data were outside the scope of this paper as they typically require integration of information from the entire genome $^{23,35}$.

Data availability. The datasets generated during and/or analysed during the current study are available from the corresponding author on reasonable request.

\section{References}

1. WHO. Information note on recommended selection criteria for procurement of malaria rapid diagnostic tests (RDTs). Geneva, Switzerland: World Health Organization (2016).

2. Baker, J. et al. Genetic diversity of Plasmodium falciparum histidine-rich protein 2 (PfHRP2) and its effect on the performance of PfHRP2-based rapid diagnostic tests. J Infect Dis 192, 870-877, https://doi.org/10.1086/432010 (2005).

3. Lee, N. et al. Effect of sequence variation in Plasmodium falciparum histidine- rich protein 2 on binding of specific monoclonal antibodies: Implications for rapid diagnostic tests for malaria. J Clin Microbiol 44, 2773-2778, https://doi.org/10.1128/JCM.0255705 (2006).

4. WHO. Malaria rapid diagnostic test performance: results of WHO product testing of malaria RDTs: round 6 (2014-2015). Geneva: World Health Organization (2015).

5. WHO. World Malaria Report 2016. Geneva: World Health Organization (2016).

6. Houze, S., Boly, M. D., Le Bras, J., Deloron, P. \& Faucher, J. F. PfHRP2 and PfLDH antigen detection for monitoring the efficacy of artemisinin-based combination therapy (ACT) in the treatment of uncomplicated falciparum malaria. Malar J 8, 211, https://doi. org/10.1186/1475-2875-8-211 (2009).

7. Wanja, E. W. et al. Field evaluation of diagnostic performance of malaria rapid diagnostic tests in western Kenya. Malar J 15, 456, https://doi.org/10.1186/s12936-016-1508-y (2016). 
8. Gamboa, D. et al. A large proportion of P. falciparum isolates in the Amazon region of Peru lack pfhrp2 and pfhrp3: implications for malaria rapid diagnostic tests. PLoS One 5, e8091, https://doi.org/10.1371/journal.pone.0008091 (2010).

9. Bharti, P. K. et al. Prevalence of pfhrp2 and/or pfhrp3 Gene Deletion in Plasmodium falciparum Population in Eight Highly Endemic States in India. PLoS One 11, e0157949, https://doi.org/10.1371/journal.pone.0157949 (2016).

10. Koita, O. A. et al. False-negative rapid diagnostic tests for malaria and deletion of the histidine-rich repeat region of the hrp2 gene. Am J Trop Med Hyg 86, 194-198, https://doi.org/10.4269/ajtmh.2012.10-0665 (2012).

11. Parr, J. B. et al. Pfhrp2-Deleted Plasmodium falciparum Parasites in the Democratic Republic of the Congo: A National Crosssectional Survey. J Infect Dis 216, 36-44, https://doi.org/10.1093/infdis/jiw538 (2017).

12. Kozycki, C. T. et al. False-negative malaria rapid diagnostic tests in Rwanda: impact of Plasmodium falciparum isolates lacking hrp2 and declining malaria transmission. Malar J 16, 123, https://doi.org/10.1186/s12936-017-1768-1 (2017).

13. Berhane, A. et al. Rapid diagnostic tests failing to detect Plasmodium falciparum infections in Eritrea: an investigation of reported false negative RDT results. Malar J 16, 105, https://doi.org/10.1186/s12936-017-1752-9 (2017).

14. Berhane A, M. S., Mohammed, S., Embaye G, H. F., Zehaie, A. \& Chinorumba A, C. J. Pfhrp2 detecting malaria rdts: Alarming false negative results in eritrea,. In: Program and Absracts of the 65th Conference on American Soceity for Tropical Medicine and Hygiene, Atlanta. 879 (2016).

15. Gatton, M. L. et al. Use of PfHRP2-only RDTs rapidly select for PfHRP2-negative parasites with serious implications for malaria case management and control. J Infect Dis, https://doi.org/10.1093/infdis/jix094 (2017).

16. Cheng, Q. et al. Plasmodium falciparum parasites lacking histidine-rich protein 2 and 3: a review and recommendations for accurate reporting. Malar J 13, 283, https://doi.org/10.1186/1475-2875-13-283 (2014).

17. Kamau, E. et al. K13-Propeller Polymorphisms in Plasmodium falciparum Parasites From Sub-Saharan Africa. J Infect Dis. https:// doi.org/10.1093/infdis/jiu608 (2014).

18. Amoah, L. E., Abankwa, J. \& Oppong, A. Plasmodium falciparum histidine rich protein-2 diversity and the implications for PfHRP 2: based malaria rapid diagnostic tests in Ghana. Malar J 15, 101, https://doi.org/10.1186/s12936-016-1159-z (2016).

19. Kemp, D. J., Thompson, J. K., Walliker, D. \& Corcoran, L. M. Molecular karyotype of Plasmodium falciparum: conserved linkage groups and expendable histidine-rich protein genes. Proc Natl Acad Sci USA 84, 7672-7676 (1987).

20. Rock, E. P. et al. Comparative analysis of the Plasmodium falciparum histidine-rich proteins HRP-I, HRP-II and HRP-III in malaria parasites of diverse origin. Parasitology 95(Pt 2), 209-227 (1987).

21. Baker, J. et al. Transcription and expression of Plasmodium falciparum histidine-rich proteins in different stages and strains: implications for rapid diagnostic tests. PLoS One 6, e22593, https://doi.org/10.1371/journal.pone.0022593 (2011).

22. Teng, X. et al. Genome-wide consequences of deleting any single gene. Mol Cell 52, 485-494, https://doi.org/10.1016/j. molcel.2013.09.026 (2013).

23. Sepulveda, N. et al. A Poisson hierarchical modelling approach to detecting copy number variation in sequence coverage data. $B M C$ Genomics 14, 128, https://doi.org/10.1186/1471-2164-14-128 (2013).

24. Githinji, S. et al. A national health facility survey of malaria infection among febrile patients in Kenya, 2014. Malar J 15, 591, https:// doi.org/10.1186/s12936-016-1638-2 (2016).

25. Malaria, G. E. N. P. f. C. P. Genomic epidemiology of artemisinin resistant malaria. eLife 5, https://doi.org/10.7554/eLife.08714 (2016).

26. Borrmann, S. et al. Genome-wide screen identifies new candidate genes associated with artemisinin susceptibility in Plasmodium falciparum in Kenya. Scientific reports 3, 3318, https://doi.org/10.1038/srep03318 (2013).

27. Cnops, L., Boderie, M., Gillet, P., Van Esbroeck, M. \& Jacobs, J. Rapid diagnostic tests as a source of DNA for Plasmodium speciesspecific real-time PCR. Malar J 10, 67, https://doi.org/10.1186/1475-2875-10-67 (2011).

28. van Schalkwyk, D. A. et al. Culture-adapted Plasmodium falciparum isolates from UK travellers: in vitro drug sensitivity, clonality and drug resistance markers. Malar J 12, 320, https://doi.org/10.1186/1475-2875-12-320 (2013).

29. Snounou, G. \& Singh, B. Nested PCR analysis of Plasmodium parasites. Methods in molecular medicine 72, 189-203, https://doi. org/10.1385/1-59259-271-6:189 (2002).

30. Snounou, G. \& Beck, H. P. The use of PCR genotyping in the assessment of recrudescence or reinfection after antimalarial drug treatment. Parasitol Today 14, 462-467, doi:S0169-4758(98)01340-4 [pii] (1998).

31. Beshir, K. B. et al. Residual Plasmodium falciparum parasitemia in Kenyan children after artemisinin-combination therapy is associated with increased transmission to mosquitoes and parasite recurrence. J Infect Dis 208, 2017-2024, https://doi.org/10.1093/ infdis/jit431 (2013).

32. Beshir, K. B. et al. Measuring the efficacy of anti-malarial drugs in vivo: quantitative PCR measurement of parasite clearance. Malar J9, 312, https://doi.org/10.1186/1475-2875-9-312 (2010)

33. Padley, D. J., Heath, A. B., Sutherland, C., Chiodini, P. L. \& Baylis, S. A. Establishment of the 1st World Health Organization International Standard for Plasmodium falciparum DNA for nucleic acid amplification technique (NAT)-based assays. Malar J 7 , 139, [pii] https://doi.org/10.1186/1475-2875-7-139 (2008).

34. Campino, S. et al. Genomic variation in two gametocyte non-producing Plasmodium falciparum clonal lines. Malar J 15, 229, https://doi.org/10.1186/s12936-016-1254-1 (2016)

35. Boeva, V. et al. Control-FREEC: a tool for assessing copy number and allelic content using next-generation sequencing data. Bioinformatics 28, 423-425, https://doi.org/10.1093/bioinformatics/btr670 (2012).

\section{Acknowledgements}

We thank the primary study participants and their parents, and village and district authorities, for their assistance obtaining samples in Mbita; Geoffrey Omondi Olweru and David John Odoyo for their contributions to the Mbita field work; Angela Hunt Cooke (MRL, LSHTM) for microscopic slide reading and Ernest Diez-Benavente (LSHTM) for helping with the access to the genomic data. We are also grateful to Qin Cheng (Australian Army Malaria Institute) for valuable advice on laboratory methods during preparatory discussions for this work. We also thank ACT Consortium, which is funded through a grant from the Bill and Melinda Gates Foundation to the London School of Hygiene \& Tropical Medicine, for sponsoring the laboratory work. The collection of samples in Mbita was supported by the Netherlands Organization for Scientific Research, division Medical Science (grant number 91211038). Fundação para Ciência e Tecnologia for partially funding N.S (grant UID/MAT/00006/2013).

\section{Author Contributions}

K.B.B., C.J.S., J.C. and H.H. contributed to study design. A.R., A.O.B., J.G.B. coordinated and conducted the field study. K.B.B., J.B., A.R. and J.M. did laboratory work. K.B.B., N.S. and A.R. analyzed the data. K.B.B., N.S., C.J.S., J.C. and H.H. wrote the manuscript. All authors contributed to and approved the final version of the manuscript. 


\section{Additional Information}

Supplementary information accompanies this paper at https://doi.org/10.1038/s41598-017-15031-2.

Competing Interests: The authors declare that they have no competing interests.

Publisher's note: Springer Nature remains neutral with regard to jurisdictional claims in published maps and institutional affiliations.

(c) (i) Open Access This article is licensed under a Creative Commons Attribution 4.0 International License, which permits use, sharing, adaptation, distribution and reproduction in any medium or format, as long as you give appropriate credit to the original author(s) and the source, provide a link to the Creative Commons license, and indicate if changes were made. The images or other third party material in this article are included in the article's Creative Commons license, unless indicated otherwise in a credit line to the material. If material is not included in the article's Creative Commons license and your intended use is not permitted by statutory regulation or exceeds the permitted use, you will need to obtain permission directly from the copyright holder. To view a copy of this license, visit http://creativecommons.org/licenses/by/4.0/.

(C) The Author(s) 2017 